\title{
Passive Mode-locked Operation of Large Mode Area fibre lasers
}

N. G. R. Broderick, H. L. Offerhaus, D. J. Richardson, J. Caplen, L. Dong

Optoelectronics Research Centre, University of Southampton, Southampton, SO1 7 1BJ, UK.

Phone: +44 (0)1703 593144, Fax: +44 (0)1703 593142, email: ngb@orc.soton.ac.uk

\section{R. A. Sammut}

Australian Defense Force Academy, University College, Canberra, ACT 2600, Australia

We report the first demonstration of passive mode-locking in large mode area fibre lasers. Pulses as short as $900 \mathrm{fs}$ with energies of $1 \mathrm{~nJ}$ have been observed. This represents a power scaling of two orders of magnitude relative to conventional fibre design. 


\title{
Passive Mode-locked Operation of Large Mode Area fibre lasers
}

\author{
N. G. R. Broderick, H. L. Offerhaus, D. J. Richardson, R. A. Sammut, J. Caplen, L. Dong \\ Optoelectronics Research Centre, University of Southampton, Southampton, SO17 1BJ, UK. \\ Phone: +44 (0)1703 593144, Fax: +44(0)1703 593142, email: ngb@orc.soton.ac.uk
}

Recently much work on fibre lasers has concentrated on improving the maximum output energy and peak powers available from such systems. An important recent advance in this area has been the development of single transverse-mode, large mode area (LMA-) doped fibre ${ }^{1}$. The LMA gives increased energy storage, reduces the impact of optical nonlinearities and improves the power handling capabilities of the fibre. We have developed LMA fibre designs allowing robust, single transverse-mode laser operation with fibre Mode Field Diameters (MFDs) in excess of $30 \mu \mathrm{m}$, i.e around 5 times larger than conventional doped fibre designs. Q-switch fibre lasers based on this fibre have yielded pulse energies in excess of $0.5 \mathrm{~mJ}$. Here we present the first demonstration of the use of such fibre in a passively mode-locked soliton laser. Subpicosecond, $1 \mathrm{~nJ}$ soliton pulses are obtained representing more than an order of magnitude increase in pulse energy relative to conventional soliton lasers.

In order to demonstrate soliton mode-locking using LMA fibres we decided to construct a frequencyshift, soliton fibre laser ${ }^{2}$. Our Fabry-Perot resonator design is shown in Fig.1. The basic mode-lock mechanism is provided by the combined effects of frequency shift (from the intracavity acousto-optic modulator), filtering (by the laser gain bandwidth) and self phase modulation within the fibre. However, the incorporation of the polariser (and waveplates) within the cavity allow for additional pulse narrowing and soliton formation due to nonlinear polarisation rotation. The laser was able to operate in two distinct mode-lock regimes depending on the polarisation control within the cavity. Without the polariser (i.e. as a simple frequency shift laser) high energy (up to 20nJ), 20ps chirped pulses were generated (see Fig.1), this pulse energy is probably the highest ever reported from a mode-locked fibre laser cavity. However, on incorporating the polariser stable, sub-picosecond soliton operation was achieved. High quality, transform-limited, $1 \mathrm{~nJ}$ soliton pulses as short as $900 \mathrm{fs}$ were obtained. From the characteristic soliton side-bands on the spectrum we determined the fibre dispersion to be $\approx 20 \mathrm{ps} /(\mathrm{nm} . \mathrm{km})$. Our measured MFD was $\approx 35 \mu \mathrm{m}$. From these pulse and fibre parameters we estimate the soliton order to be 1.24 at the laser output. This value is entirely reasonable and further substantiates our MFD measurement. The fundamental soliton energy in a conventional doped fibre would be $\approx 20 \mathrm{pJ}$.

In conclusion, we have successfully generated nJ pulses from a passively mode-locked, LMA fibre laser and confirmed the expected energy (power) scaling with increased spot size. This scaling suggests that energies well in excess of $0.2 \mu \mathrm{J}$ should be achievable using a different mode-locked cavity design.

1. D. J. Richardson et al., Elect. Lett., 33 (23) 1955-1956 (1997)

2. M. Romagnoli .et al. J. Opt. Soc. Am. B, 12(1) 72-76 (1995)
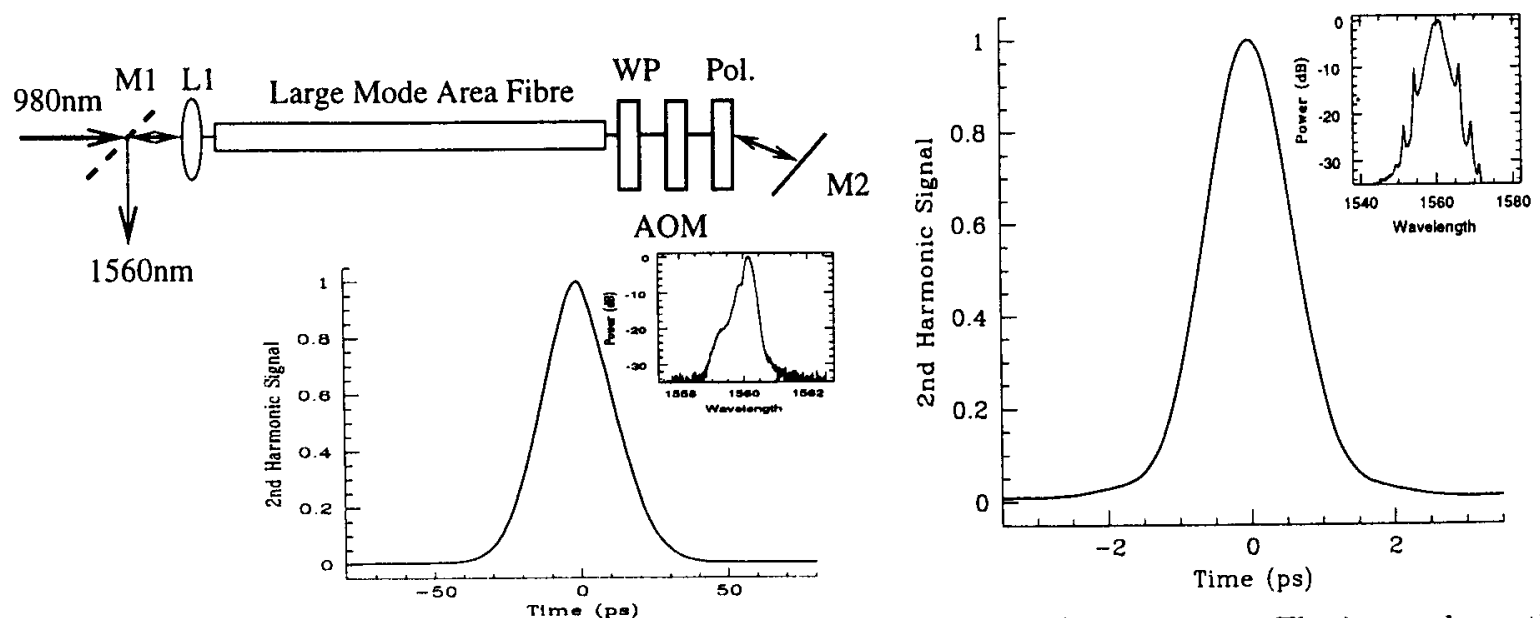

Fig. 1. (a)Experimental Setup: $\mathrm{M} 1$-dichroic mirror, WP: $\lambda / 2$ and $\lambda / 4$ waveplates. The insert shows the autocorrelation trace and pulse spectrum corresponding to a pulse width of $20 \mathrm{ps}$. (b) Autocorrelation and pulse spectrum for the $900 \mathrm{fs}$ pulses; the spectrum shows distinctive sidelodes common to soliton lasers. The pulse energy is approximately $1 \mathrm{~nJ}$. 